\title{
DYNAMIC EVALUATION OF THE USEFUL FIELD OF VIEW IN DRIVING
}

\author{
Daniel R. Mestre \\ Cognitive Neurosciences Centre \\ Centre National de la Recherche Scientifique (CNRS) \\ Marseilles, France \\ E-mail: mestre@lnf.cnrs-mrs.fr
}

\begin{abstract}
Summary: The concept of "useful field of view " (UFOV) was introduced to describe the area from which useful visual information can be extracted in a single glance. It is not restricted to the fovea, nor does it involve the entire visual field. It is often claimed that the UFOV decreases with age, with increasing speed of travel, or under the influence of drugs or stress. However, this potentially important tool for the evaluation of the role of human sensorial factors in road safety suffers from a lack of measurement techniques. Within this framework, we evaluated the ability of human observers to discriminate variations in their direction of heading from optical flow patterns simulating self-motion relative to a simple toric surface (a curved "tunnel"). We systematically evaluated perceptual performance as a function of the part of the global optical flow observers were looking at. Inasmuch as experimental laboratory data can be generalized to the complex task of driving, the results suggest that the perception of heading is optimal in a limited part of the visual field, situated around the future direction of travel. They offer a novel approach to the concept of useful field of view. They can be discussed in terms of their implications for road infrastructure design and for the positioning of warning and traffic signs within the driver's dynamic visual environment.
\end{abstract}

\section{THE ROLE OF VISION IN DRIVING}

If one accepts that the information input to the typical driver is mainly visual (Hills, 1980), the problem remains to determine which visual cues are involved, depending on the actual sub-task of the overall driving process (i.e. highway cruising vs. intersection crossing), and on the internal state of the driver (i.e. his/her age, experience in driving, level of awareness, and other human factors). One way to tackle this problem has been to look for correlations between the driver's visual performance and accident rate, involving large-scale investigations.

In 1968, Burg reported the results of a large-scale study, involving visual measurements in Californian drivers. To provide driver-licensing administrators with here-to-fore unavailable information on which to establish effective vision-screening procedures for driver license applicants, a number of visual performance, personal, and driving habit characteristics of some 17,500 volunteer California driver license applicants were compared with their 3-year driving records (accidents and convictions). Of all the visual tests, dynamic visual acuity was most closely and consistently correlated with driving record, followed by static acuity, field of view, 
and glare recovery. All relationships were in the "expected" direction, i.e., poor vision was associated with poor driving records. We want here to spend some time presenting data on the concept of field of view, and more precisely the useful field of view.

\section{THE CONCEPT OF USEFUL FIELD OF VIEW}

The useful field of view (UFOV) is defined as the visual area from which information can be acquired without moving the eye or turning the head (Ball et al., 1988). The concept of UFOV was developed from more classical perimetric studies of the visual field in humans. A number of reports argued for a reduction in the size of the visual field as a function of age. However, perimetric tests of the visual field (figure 1), although efficient diagnostics of disease, underestimate the degree of difficulty experienced by visually healthy older adults in everyday activities requiring the use of peripheral vision.

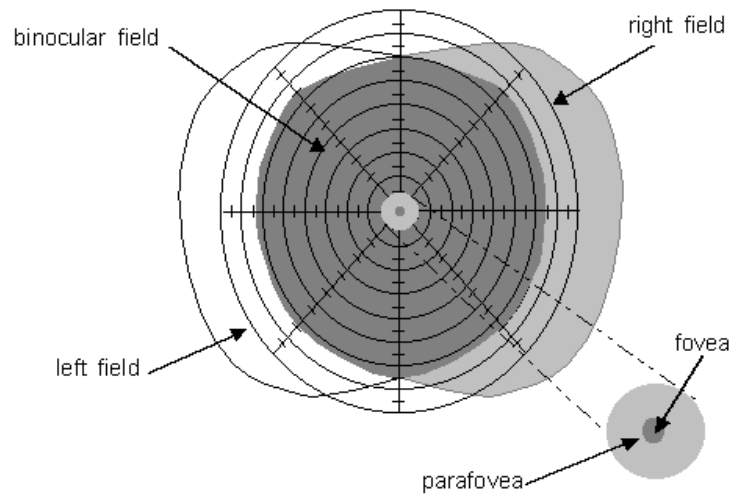

Figure 1. The complete visual field in humans, extending more than 180 degrees in the horizontal dimension. The fovea is the area (about 3 deg in diameter) in which visual acuity is highest. The parafovea (about $10 \mathrm{deg}$ ) is the zone is which some pre-processing of visual inputs (like during reading) occurs. The rest of the visual field is called peripheral (low visual acuity, good temporal resolution)

In order to develop a more functional approach, a model incorporating the effects of peripheral distractors and secondary tasks on a primary foveal task demands was developed (Ball et al., 1988). Subjects responded to dual tasks, one central, one peripheral (10 to $30 \mathrm{deg}$ in eccentricity). Using such methodology, experimental studies demonstrated that the size of the UFOV was more flexible than the physiologically determined size of foveal and parafoveal zones. The size of the UFOV is inversely correlated to the foveal task demand, is related to accident risk experienced notably by elderly drivers, in which its size is reduced, as compared to young drivers (Ball et al., 1993). It is also sensitive to (expending with) driving expertise (Crundall et al., 1999).

\section{OPTIC FLOW}

In the logic presented above, researchers try to evaluate the size of the useful field of view, with reference to the observer's central point of fixation. However, we wish to mention here that there 
are reasons to suspect that the direction of gaze, while driving, is an important matter in itself. More precisely, this question refers to an important ongoing debate in the scientific community: Where do you look when you drive? (Land \& Lee, 1994).

This problem brings us back to the approach to the problem of the visual basis for the control of locomotion, formalized by Gibson (1979), who introduced the concept of optic flow, to describe the transformations of the optic array projected onto the entire retina during self-motion. In an "interactionnist" approach, he suggested that our motion through the environment produces a pattern of optic flow, which specifies the properties of our displacement (figure 2).

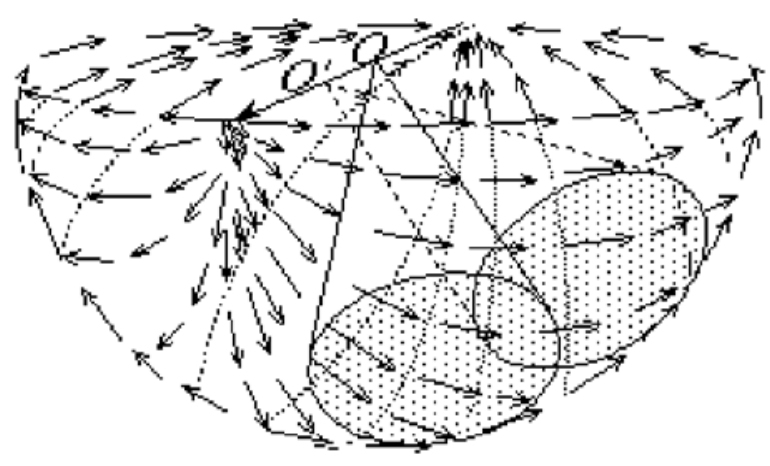

Figure 2. The optic flow field is described as a two-dimensional motion field, specifying the direction of travel of the observer $(\mathrm{O})$, here parallel to the ground surface. Gibson proposed that the information was present everywhere in the optic flow field (i.e. irrespective of the moving observer's direction of gaze).

In the 90's, Warren and collaborators (Warren et al., 1988, 1991) demonstrated that heading perception from optic flow accuracy was of the order of 1 degree for both rectilinear and curvilinear paths of self-motion. The main thesis, originating from Gibson himself, was that perception relied on the global optic flow pattern.

However, Land and Lee (1994) showed that, in curve negotiation, the eyes tend to fixate the inside edge of the road near a point known as the "tangent" or "reversal" point of the road, which is a point where the inside of the curve changes direction (Figure 3). This suggests that subjects pick up useful information there. 


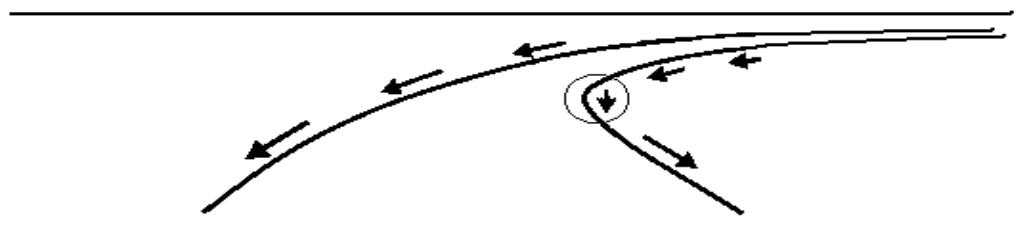

Figure 3. While negotiating a curve, drivers tend to fixate a region (circled) where the inside edge of the road changes direction. This is also the point where the horizontal component of the optical motions of the road markers changes direction (from leftward to rightward in this case).

\section{EXPERIMENTAL WORK}

Within this framework, we set up an experimental study, aimed at evaluating the ability of (young) human observers to perceive their direction of travel from optic flow patterns, as a function of their direction of gaze in a virtual environment. We simulated curvilinear self-motion inside a simple toric surface (figure 4). Using random-dot optic flow stimuli of brief duration and a two-alternative forced-choice adaptive procedure, we evaluated curvature discrimination thresholds in healthy volunteers, as a function of the direction of gaze.

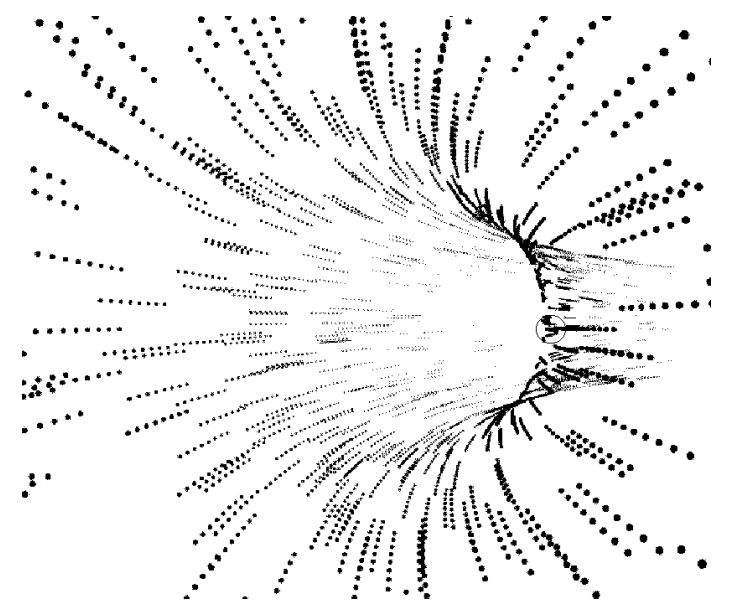

Figure 4. Time-exposure slide, representing the optic flow that subjects experienced during testing. The superimposed circle represents the tangent point of the random-dot tunnel. At the tangent point, the optical velocity is null, whenever the trajectory of the observer is perfectly aligned with the tunnel. In the experiment, subjects had to detect the situation where their radius of self-curvature was higher than the (constant) radius of the tunnel.

Results clearly indicate that curvature discrimination thresholds are always minimal when subjects' direction of gaze is aligned with the tangent point. As the direction of gaze goes 
towards eccentric parts of the optic flow field (relative to the tangent point), thresholds increase significantly. The pattern of evolution is coherent with a local analysis of optic flow velocities (figure 5).

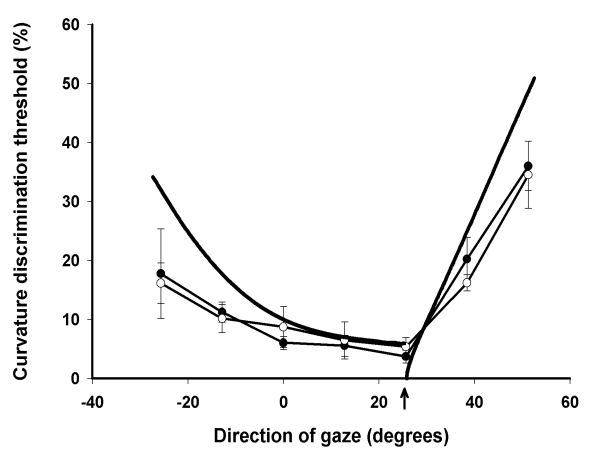

Figure 5. Curvature discrimination thresholds (expressed as a percentage of the tunnel's radius of curvature) in two observers, as direction of gaze departures from the tangent point of the driving environment (vertical arrow). The bold line represents the outcome of a model predicting discrimination thresholds from local velocity discrimination in foveal vision.

\section{CONCLUSION}

The present results show that the visual perception of the spatial characteristics of self-motion is not equally precise throughout the visual field. They indicate that spontaneous gazing strategies observed during driving might correspond to an optimal selection of useful information in the optic flow field. We believe that such data could be beneficially incorporated in current approaches of the useful field of view. In other terms, current approaches of the useful field of view evaluate peripheral processing of visual input in egocentric terms (i.e. relative to a "foveal" task). Here, we show that the direction of gaze itself (i.e. the location of the fovea) of the moving observer is dependant on the structure of the optic flow field, being dependant itself on the environmental structure. There are thus egocentric and exocentric determinants of the useful field of view. This approach to the problem of the driver's dynamic visual environment might have implications, concerning not only the evaluation of driving performance in different populations, but also the architecture of the driver's visual environment for safe driving. For instance, highway engineers might want to avoid attracting the driver's gaze toward eccentric zones of the dynamic visual environment.

\section{ACKNOWLEDGMENTS.}

This research was supported by CNRS and by a grant from the Ministry of Research and Technology (PREDIT 98 T 0018). 


\section{REFERENCES}

Ball, K. K., Beard, B. L., Roenker, D. L., Miller, R. L.,Griggs D. S. (1988). Age and visual search: expanding the useful field of view. Journal of the Optical Society of America A, 5(12) 2210-2219.

Ball, K., Owsley, C., Sloane, M.E., Roenker, D.L., Bruni, J.R. (1993). Visual attention problems as a predictor of vehicle crashes in older drivers. IOVS, 34(11), 3110-3123.

Burg, A. (1968). Vision and driving: A summary of research findings. Highway Research Record, Number 216, 1-12.

Crundall, D., Underwood, G., Chapman, P. (1999). Driving expertise and the functional field of view. Perception, 28, 1075-1087.

Gibson, J.J. (1979). The Ecological Approach to Visual Perception. Boston: Houghton Mifflin.

Hills, B.L. (1980). Vision, visibility and driving. Perception, 9, 183-216.

Land, M., Lee, D.N. (1994). Where we look when we steer. Nature, 369, 742-744.

Warren, W.H. \& Hannon, D.J. (1988). Direction of self-motion is perceived from optical flow. Nature, 336, 162-163.

Warren, W.H., Mestre, D.R., Blackwell, A.W. \& Morris, M.W. (1991). Perception of circular heading from optical flow. Journal of Experimental Psychology: Human Perception and Performance, 17, 28-43. 